\title{
Use of Holographic Technology in Online Experimentation
}

\author{
Jakub Matišák \\ Slovak University of \\ Technology, Faculty of Electrical \\ Engineering and Information \\ Technology, Ilkovičova 3, 81219 \\ Bratislava, Slovakia \\ Email: jakub.matisak@stuba.sk
}

\author{
Matej Rábek \\ Slovak University of \\ Technology, Faculty of Electrical \\ Engineering and Information \\ Technology, Ilkovičova 3, 81219 \\ Bratislava, Slovakia \\ Email: matej.rabek@stuba.sk
}

\author{
Katarína Žáková \\ Slovak University of \\ Technology, Faculty of Electrical \\ Engineering and Information \\ Technology, Ilkovičova 3, 81219 \\ Bratislava, Slovakia \\ Email: katarina.zakova@stuba.sk
}

\begin{abstract}
The paper deals with a web application that allows simulating 3D model of mechatronic system in holographic devices. Purpose of this application is to bring new perspective to interactive education and simplify the process of studying. Background of an application is driven by Scilab Xcos simulation software communicating via web service, which provides data. The resulting animation is displayed on a holographic device, which allows visualization. The displayed system is a 3D model of the mechatronic experiment, which represents digital model of real device. Accurate movement of experiment is obtained by linking data from Scilab Xcos with 3D model. The 3D model visualization should help with easier understanding of the subject matter.
\end{abstract}

Keywords - mechatronic system, simulation, animation, holographic technology

\section{INTRODUCTION}

$\mathrm{T}_{\mathrm{i}, \mathrm{s}}^{\mathrm{H}}$ HE goal of each educational institution should be to improve and innovate the educational process. New methods of educating students should be formed to make the process easier, such as in [1]. Also, it is necessary to increase its efficiency. The ideal outcome is to bring education and research activities together, creating innovations that support the industry [2]. The latest trend in education is to bring an application that gives a better understanding of the issue. Simplifying device designs, understanding technical specifications, facilitating device prototyping, or even making manufacturing process cheaper are just a few of the many different uses of 3D hardware digitalization [3]. There are many three-dimensional environments around the world that try to incorporate, work, and simulate knowledge from different areas [4]. Study says that at laboratory sessions 58\% students agreed that methodologies like simulations, demonstrations and virtual labs make them more comfortable in lab sessions [5]. Nowadays, we can observe the trend of digitizing [6] and simulating equipment in almost every working segment. It allows us to face real situations before they happen, to learn from them, see issues from another perspective, respond to them much faster and, finally, to save costs. Lately many institutions have specialized in virtual and augmented reality, like in [7] and brought attention to it. However, our project wants to focus on an area that is not so widespread. The aim of our work is to show students another angle of learning. To do so, we used holographic technology.
Optical holography for recording three-dimensional scenes can be traced back to the early sixties. Since then, the art of holography has been applied in many areas, primarily as a tool for 3D imaging, processing, and display [8]. Study in [9] says that $45.5 \%$ of teachers believe that hologram technology would have affect in the field of teaching. The use of holographic technology could be used in various areas of life. The first example is using it in car, which is published in [10]. The authors attempt to present a holographic display, that would help reduce the time when drivers were guided to the dashboard. Hologram would be projected onto the front glass, so time of inattention would be reduced. Another example is in medicine. There is a possibility of displaying real heart beating on a model of heart in four-sided hologram pyramid, which authors published in [11].

The aim of this paper is to help students with understanding of the subject matter dealing, for instance with the basics of automatic control. Our system can simply help to visualize the behavior of mechatronic experiment parts as 3D digital model in holographic device.

\section{State Of TeChNOLOGY}

The "Hologram" word refers to a three-dimensional picture made by laser light reflected onto a photographic substance without the use of a camera [12]. Hologram device could be used to play video, represent some system behavior, show object models, etc. We know many varieties of holograms, and there are variable ways of classifying them. For our purpose, we can divide them into three main types: reflection, transmission and hybrid holograms.

\section{A. Reflection holograms}

The reflection hologram (Fig. 1) is the most common type of the hologram. They can be seen in galleries and in presentation places. This hologram is formed when the reference beam and the object beam are incident on opposite sides of the holographic surface. They interfere and record an image. To reconstruct the image, a point source of white light illuminates the hologram from the proper angle, and the viewer looks at it from the same side as the light source. Reflection holograms require the simplest setup and are visible without laser light [13]. 


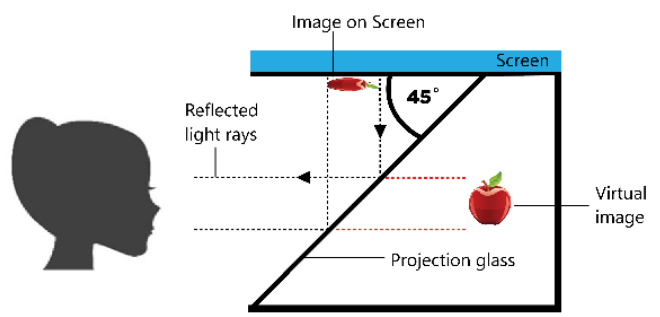

Fig. 1 How reflection hologram works

\section{B. Transmission holograms}

Transmission holograms (Fig. 2) are also known as LaserTransmission Holograms. This type is created when the reference beam and the object beam are incident on the same side of the holographic surface. They are viewed by shining a spread-out laser light through the emulsion side of the hologram at the same angle the hologram was recorded at, with the viewer looking on from the opposite side. The light is transmitted from behind the hologram device to the side of the observer [13]. Image which is displayed can be very precise.

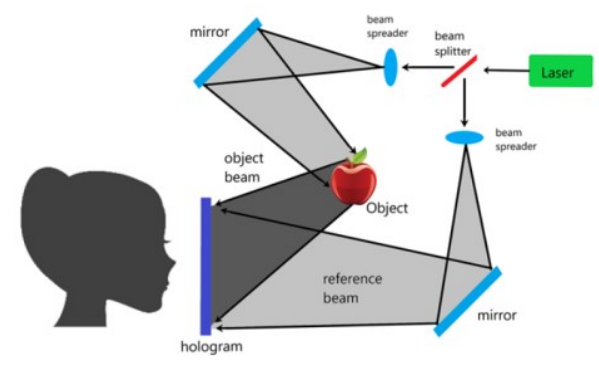

Fig. 2 How transmission hologram works

Materials, methods, and processes used to make transmission hologram are the same as reflection holograms.

\section{Hybrid holograms}

As hybrid hologram could be considered a combination of transmission and reflection hologram. Hybrid hologram can be specified as multichannel holograms, holographic interferometry, integral holograms, embossed holograms, and computer-generated holograms. For example, embossed holograms are used for authenticity applications such as security hologram stickers, passports or credit cards. Computer-generated holograms are used to make optical elements, for scanning, splitting, in general for controlling laser light, example can be CD player [14]. These types of hologram are not relevant for our work, because of their technology, so we will not pay attention to them.

\section{PRODUCT RESEARCH}

Looking on the market the most advanced holographic device suitable for our purpose can be considered Microsoft HoloLens. HoloLens is a pair of mixed reality smart glasses, which is a holographic computer built into a headset that lets you see, hear, and interact with holograms within an environment such as a living room or an office space [15]. It is a wearable device that permits to look at holograms that are connected to the world and interact with them using gestures, voice commands and gaze. For instance, one of its advantages is plugin for Unity engine. However, due to its higher price (3500\$), it is unlikely that will be massively adapted soon.

Next, the Realfiction company offers a range of holographic devices. These devices have different sizes and differences in the number of display areas. They also have higher prices $(2000 \$-10000 \$)$. In our project we approached to use the Realfiction Dreamoc HD3.2. Device has threesided view, HDMI port, RJ45 port 23" screen and built-in loudspeakers. More details can be found in [20]. We chose it because this device has connectivity advantage - HDMI.

For everyday use it is possible to buy quite cheap holographic devices and use it with smartphones or tablets for example from company Holho. Price can be from $40 \$$ to $160 \$$. Disadvantage of these devices is that only video can be played, so when we want to control behavior of real-time experiment it is not possible to change anything in process without connection to another device via cable.

\section{APPLICATION}

We had following requirements for the application:

- to make realistic view of 3D mechatronic experiment,

- to control the behavior of the 3D model using parameters entered by the user.

\section{A. Hardware}

As it was said before, it's necessary to have hardware to generate holographic image. This hardware consists of two main parts. The device from the front size is shown in Fig.3.

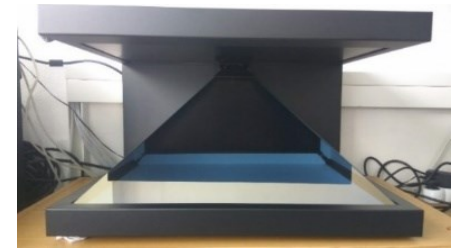

Fig. 3 Hologram device from the front side

The first part is the image-emitting screen. A conventional computer screen could be used, but the resolution of screen increases the quality of the image. Our device has a screen up and emits the image from top to bottom. The second part is a projection glass with a semi-permeable layer, which is placed at a 45-degree angle below the screen. Therefore, the screen provides an image that is reflected on the glass. By placing the glass at this specific angle, the image is presented as if it is behind the glass, which creates virtual image of the represented object.

\section{B. $\quad$ Software and system architecture}

Application with minimal requirements and possibility of massive adoption in future led us to use standard web technologies (HTML, CSS, JavaScript and Three.js). It is necessary to realize that the display area is in the hologram, so it is not possible to change the control parameters from there. To do so, we needed to use two browser windows. The 
first one is opened in hologram as a view and the second one in computer screen as a control window. It is allowed via multi-screen mode. Of course, whole system is more complicated, and its architecture is shown in Fig.4.

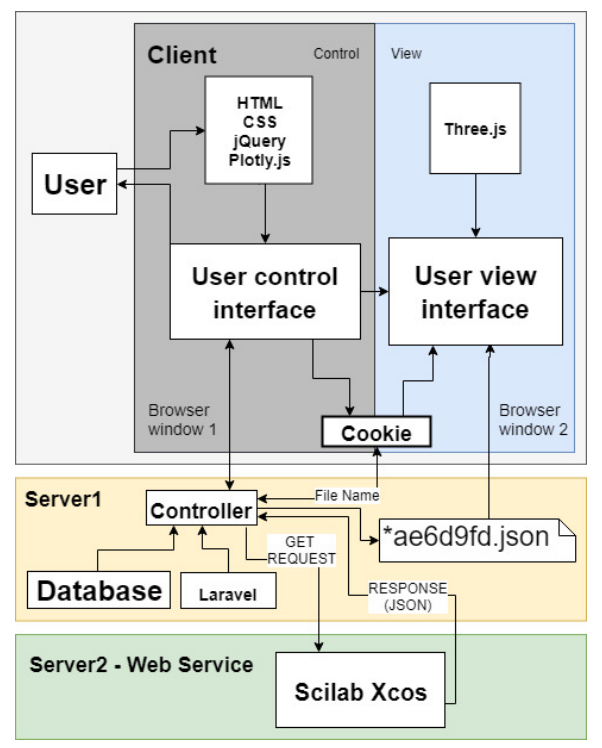

Fig. 4 System architecture

Back-end side of the application is managed from the server using a Laravel framework. When control interface is opened, data for specific experiment are automatically downloaded from MySQL database and a HTML form is generated. This form is generic, to ensure the possibility of adding new experiments in the future. The realistic movement visualization can be achieved thanks to numerical data received from Scilab Xcos simulation environment where the experiment is represented as a block diagram. Scilab allows to simulate a block scheme by accessing it through a terminal. Module with Scilab has its own interface, works as standalone application and it is used as a web service. It is necessary upload block scheme to the server before the first simulation. Then, it is used by an authorized program, accessed via URL. The main problem of showing data in $3 \mathrm{D}$ model is to send them from control to view interface or to change the simulation parameters during the visualization. Since each client window works separately, it is necessary to inform view interface about parameter changes in control interface. Solution we used is simple, when control view is opened, random SHA code is generated and saved as cookie value. This cookie is used then as a file name for data from simulation. Data are sent as a HTTP response from service and always saved to this specific file during entire session. User view interface gets these data and starts to render movement of experiment.

\section{Web Service}

There are several simulation programs on the market, like Matlab, Scilab Xcos or Octave. In our case, we have chosen Scilab Xcos, which is an open source distribution modeling and simulation software for numerical computation. The choice was done from several reasons. Matlab as the most used software requires license and not everyone have access to it. Therefore, it was not suitable for this implementation. Octave from the open source category does not provide a block diagram option, which is suitable for easier creation of controllers. There exist also other programs that have a graphical editor for building block diagrams and have also appropriate numerical methods to solve differential equations. However, Scilab Xcos is the closest open source option to Matlab.

Secondly, since we want the system to be modular, it is very useful to have an Application Programming Interface (API) that allows us to use this simulation software as a web service. The advantage of the simulation environment that is located on the server and works as a web service is that everyone who requires to retrieve the data needs to know only the URL and how to access the software through the appropriate API. We have already had API for Scilab Xcos at the time of implementation, so we decided to use it. Since the entire data processing module is running as a web service, it can be easily replaced by other software. In this way the application can be expanded by a different simulation environment in the future.

\section{ONLINE EXPERIMENT}

In Fig.7 a model of the Furuta pendulum for our holographic device is depicted. The holographic device we used renders three-side projection, so it is necessary to have the model displayed three times and rotated by ninety degrees. The one on the bottom is then shown on the front side of the device and the other two on each side.

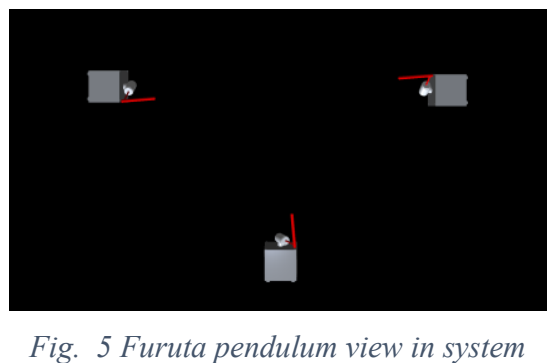

Firstly, it is necessary to open view interface (Fig.5) in holographic device.

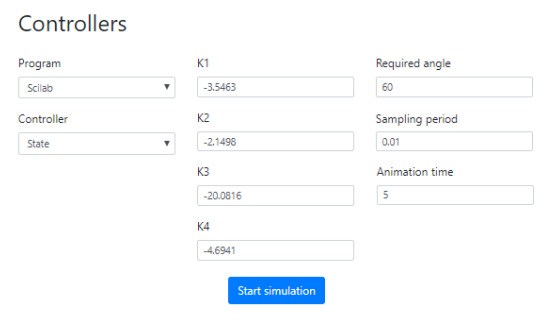

Fig. 6 Control interface for input data

Secondly, data are required. They are coming from control interface where the block scheme is simulated. In our case the control structure includes model of Furuta pendulum and the state space controller. Its parameters (i.e. gains $K 1, K 2, K 3$, $K 4$ ) can be defined via user interface shown in Fig.6. User can specify also required angle, sampling period and animation 
time. After sending the request to the server (by clicking on the button Start simulation), the data will arrive within seconds and the model simulation will automatically start. The input parameters can be altered by sending a new request. The process is designed to change movement automatically. In Fig. 7 is shown holographic device Dreamoc HD3.2 with connected computer via HDMI.

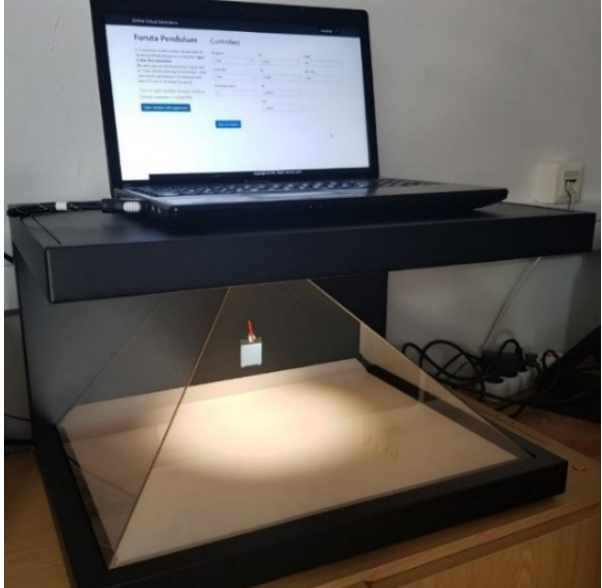

Fig. 6 Control interface in computer screen and Furuta pendulum model in holographic device

\section{Conclusion}

The paper describes web application for holographic devices, developed mostly for educational purposes. It can be used as a tool for interactive teaching in subjects dealing with automatic control theory. With this technology, for students it is simpler to imagine mechatronic systems behavior and that should be goal of every educational institution. The application acts as simulation software for 3D objects that helps students to understand how to operate with specific devices and systems. The application uses Scilab Xcos as simulation environment for computing block schemas. Achieved simulation data allow to see realistic movement of experiment 3D model. The 3D model, which is shown as holographic mechatronic experiment projection of the specific device. Holographic device that was used to show model and the behavior of the experiment, cannot be considered as a solution that could be used massively, due to its higher price. The tool can used as a display device for teachers during lessons. However, the application can be simply modified to a device that is not subject of a high price and this version could be deployed for every student.

As a future work, authors would like to extend the application by directly embedding it into the online laboratory portal, which deals with real-time control of experiments. This portal is currently in the implementation phase. Also, they would like to extend the application to use it through augmented reality, using Google ARCore platform.

ACKNOWLedgment

The work presented in this paper has been supported by the Cultural and Educational Grant Agency of the Ministry of
Education, Science, Research and Sport of the Slovak Republic, KEGA 030STU-4/2017 and by the Tatra Banka Foundation within the grant program E-talent, project No. 2018et016 (Holographic technology and augmented reality in online experimentation). Authors would like to thank to all contributors for help with implementation.

\section{REFERENCES}

[1] B. Kraut a J. Jeknić, „Improving education experience with augmented reality (AR)," rev. 2015 38th International Convention on Information and Communication Technology, Electronics and Microelectronics (MIPRO), Opatija, Croatia, 2015. doi:10.1109/ MIPRO.2015.7160372

[2] J. Autiosalo, „Platform for industrial internet and digital twin focused education, research, and innovation: Ilmatar the overhead crane," rev. 2018 IEEE 4th World Forum on Internet of Things (WF-IoT), Singapore, Singapore, 2018. doi:10.1109/WF-IoT.2018.8355217

[3] K. Źáková a M. Hók, „Interactive three dimensional presentation of Segway laboratory model,“ rev. 2016 International Conference on Emerging eLearning Technologies and Applications (ICETA), Vysoke Tatry, Slovakia, 2016. doi:10.1109/ICETA.2016.7802064

[4] J. Lebiedź a M. Szwoch, „Virtual sightseeing in Immersive 3D Visualization Lab," rev. 2016 Federated Conference on Computer Science and Information Systems (FedCSIS), Gdansk, Poland, 2016.

[5] M. Deepak, T. Sandeep, B. Shruti, B. Manish a S. Sneha, „An analysis to find effective teaching methodology in engineering education,“ Jaipur, India, 2013. doi:10.1109/MITE.2013.6756331

[6] B.-M. Block, ,Digitalization in engineering education research and practice," rev. 2018 IEEE Global Engineering Education Conference (EDUCON), Tenerife, Spain, 2018. doi:10.1109/EDUCON.2018. 8363342

[7] E. Kucera, O. Haffner a R. Leskovský, „Interactive and virtual/mixed reality applications for mechatronics education developed in unity engine," rev. 2018 Cybernetics \& Informatics (K\&I), Lazy pod Makytou, Slovakia, 2018. doi: 10.1109/CYBERI.2018.8337533

[8] Peter Wai Ming Tsang; Ting-Chung Poon;, „Review on the State-ofthe-Art Technologies for Acquisition and Display of Digital Holograms,“ IEEE Transactions on Industrial Informatics, zv. Volume: 12, \%1. vyd.Issue: 3, pp. 886 - 901, June 2016. doi:10.1109/ TII.2016.2550535

[9] H. Ghuloum, ,3D Hologram Technology in Learning Environment,“ rev. Proceedings of Informing Science \& IT Education Conference (InSITE) 2010, Cassino, Italy, 2010.

[10] W. Wang, X. Zhu, K. Chan a P. Tsang, ,Digital Holographic System for Automotive Augmented Reality Head-Up-Display,“ rev. 2018 IEEE $27^{\text {th }}$ International Symposium on Industrial Electronics (ISIE), Cairns, QLD, Australia, 2018. doi:10.1109/ISIE.2018.8433601

[11] T. Thap, Y. Nam, H. Chung, J. Lee, „Simplified 3D Hologram Heart Activity Monitoring Using a Smartphone," rev. 2015 9th International Conference on Innovative Mobile and Internet Services in Ubiquitous Computing, Blumenau, Brazil, 2015. doi:10.1109/IMIS.2015.87

[12] A. Abdullah H a K. Faten F., „The first design of a smart hologram for teaching," rev. 2018 Advances in Science and Engineering Technology International Conferences (ASET), Abu Dhabi, United Arab Emirates, 2018. doi:10.1109/ICASET.2018.8376931

[13] K. Fey, „Creation of Simple Holograms with Single Beam Setup,“ August 2000. [Online]. Available: https://laser.physics.sunysb.edu/ karl/webreport/index.html. [Cit. 6 May 2019].

[14] A. Jeong; T. H. Jeong;, ,What Are the Main Types of Holograms?“” Integraf LLC, 2019. [Online]. Available: https://www.integraf.com/ resources/articles/a-main-types-of-hologram s. [Cit. 6 May 2019].

[15] J. Roberts, „What is HoloLens? Microsoft's holographic headset explained,“ "Trusted Reviews, 30 March 2016. [Online]. Available: https://www.trustedreviews.com/opinion/hololens-release-date-newsand-price-2922378. [Cit. 6 May 2019].

[16] Realfiction, „Dreamoc Fact-sheet,“ [Online]. Available: https:// www.dropbox.com $/ \mathrm{s} / \mathrm{x} 7 \mathrm{n} 1 \mathrm{gvkh} 2 \mathrm{yfmyf} 3 /$ dreamochd_factsheet version-1.pdf?dl=0. [Cit. 1 May 2019]. 